\title{
Un ejemplo de educación financiada mediante criptomoneda: la ICO de la IEBS Business School
}

\author{
Raúl Jaime Maestre \\ Director del Máster en Blockchain y Fintech en la IEBS Business School, \\ profesor en la Universitat Oberta de Catalunya y doctorando en Ciencias de la Computación \\ en la Universidad Internacional de La Rioja \\ rauljaime@gmail.com
}

\section{Extracto}

Este estudio es un análisis de una empresa que trabaja en el sector de la educación y quiere ampliar su negocio a nuevos campos dentro de la formación innovadora. La IEBS Business School, la empresa que estamos analizando, quiere explotar las ventajas que ofrece la tecnología Blockchain. Por este motivo, se analizan algunas de las características principales de dicha tecnología. Se introduce al lector en el procedimiento de financiación a través de la oferta inicial de moneda (initial coin offering [ICO]), un concepto nuevo en el mundo financiero actual.

En resumen, la IEBS Business School será nuestro ejemplo de cómo el uso de la tecnología Blockchain, junto con una iniciativa de financiación a través de la ICO, puede ayudar a crear una ventaja competitiva para una empresa que tiene como objetivo expandir su negocio en el sector educativo.

Palabras clave: Blockchain; IEBS Business School; criptomoneda; token; Fintech; oferta inicial de moneda (initial coin offering [ICO]).

Cómo citar: Jaime Maestre, R. (2020). Un ejemplo de educación financiada mediante criptomoneda: la ICO de la IEBS Business School. Tecnología, Ciencia y Educación, 15, 143-163. 


\section{An example of cryptocurrency funded education: the ICO of IEBS Business School}

Raúl Jaime Maestre

\section{Abstract}

This study is an analysis of a company that works in the education sector and wants to expand its business to new fields within innovative training. IEBS Business School, the company we are analyzing, wants to exploit the advantages offered by Blockchain technology. For this reason, some of the main features of Blockchain technology are analyzed. The reader is introduced to the financing procedure through initial coin offering (ICO), a new concept in today's financial world. In summary, IEBS Business School will be our example of how the use of Blockchain technology, together with a financing initiative through ICO, can help create a competitive advantage for a company that aims to expand its business in the education sector.

Keywords: Blockchain; IEBS Business School; criptomoneda; token; Fintech; initial coin offering (ICO). 


\section{Sumario}

1. Introducción

1.1. Entender la tecnología Blockchain

1.2. Ventajas y desventajas de la tecnología Blockchain

1.3. Blockchain: una tecnología a prueba de manipulaciones

1.4. Beneficios de la tecnología Blockchain en educación

2. ¿Qué es una ICO?

2.1. ¿Cómo funciona una ICO?

2.1.1. Diferencias entre la ICO y una OPV

2.2. Ventajas y riesgos de lanzar una ICO respecto a otras fuentes de financiación

3. El caso de la IEBS Business School

3.1. El problema y la solución propuesta

3.2. Roadmap de la IEBS Business School

3.3. Venta de tokens

3.3.1. Distribución del token de la IEBS Business School

4. La importancia de la tecnología Blockchain para la IEBS Business School

5. Conclusiones

Referencias bibliográficas 


\section{Introducción}

Uno de los pilares principales de las sociedades innovadoras es la educación de sus ciudadanos. En la actualidad, vivimos en un mundo en el que la tecnología se ha convertido en el eje de nuestras vidas.

Hay una gran demanda de profesionales con formación en ciencias, tecnología, ingeniería y matemáticas, ya que las carreras vinculadas con estas materias son las que desarrollan la tecnología. Las empresas no son una excepción a las innovaciones tecnológicas. De hecho, como siempre sucede, el mundo de las empresas explota los avances tecnológicos para tratar de conseguir beneficios y satisfacer las necesidades y peticiones de sus clientes.

El caso de estudio que aquí se presenta versa sobre el desarrollo de una empresa tecnológica (Fintech) en el ámbito educativo. Fintech es un nuevo término que ha sido creado para describir el nuevo mundo de la tecnología financiera. Este nuevo espacio incluye cualquier novedad dentro del sector financiero. $Y$ varias de las mayores innovaciones en el mundo de la empresa son la tecnología Blockchain y las criptomonedas.

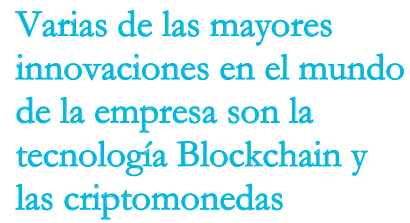

Es importante entender estos conceptos y sus implicaciones antes de explicar el proceso de la ICO. De hecho, durante esta introducción, se prepara al lector para comprender el proceso de la ICO al tratar las características principales de Blockchain. En este trabajo se intenta familiarizar al lector con los conceptos básicos de la tecnología Blockchain y con los mecanismos de una ICO. El caso de estudio utilizará una empresa en particular para mostrar cómo las nuevas tecnologías están irrumpiendo en los mercados financieros a través de la cadena de bloques y de la ICO.

En resumen, en este artículo nos vamos a centrar en la tecnología Blockchain aplicada en la educación. La tecnología Blockchain es relativamente nueva y la aplicación de la cadena de bloques en la educación es extremadamente nueva. El objetivo es analizar la tecnología Blockchain en un caso de estudio para ver algunos aspectos de mejoras que se pueden aplicar a la educación.

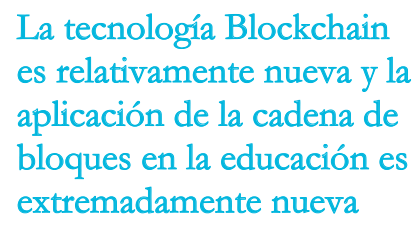

La tecnología Blockchain es relativamente nueva y la aplicación de la cadena de bloques en la educación es extremadamente nueva 


\subsection{Entender la tecnología Blockchain}

Actualmente, la tecnología Blockchain está en la mente de muchas personas. De hecho, desde los profesionales hasta el ciudadano de a pie muestran algún tipo de interés y les sorprende cuando hablamos de ella. La tecnología Blockchain se considera la clave para resolver problemas de escalabilidad, privacidad y confiabilidad (Malviya, 2016).

En 2009, se lanzó el primer bloque de una cadena de bloques a través de la tecnología Blockchain (Nakamoto, 2008). Esta criptomoneda se llama bitcoin y también es una de las principales criptomonedas a día de hoy. Una criptomoneda es una moneda digital que se ejecuta en una cadena de bloques.

Por eso se pueden explicar ambos términos de forma conjunta, porque están interconectados entre sí. La cadena de bloques que sustenta la criptomoneda bitcoin es un registro público de cada transacción que se ha realizado (Nakamoto, 2008). No puede ser manipulado o cambiado. Por esta razón, los partidarios de esta tecnología afirman que las transacciones de bitcoins son más confiables que las tradicionales.

En resumen, una cadena de bloques es un libro de contabilidad digital distribuido que permite el registro y el intercambio de información por parte de una comunidad en la que cada miembro guarda su propia copia de la información y debe validar colectivamente cualquier actualización

\section{Una cadena de bloques es un libro de contabilidad digital distribuido} (Piscini, Guastella, Rozman y Nassim, 2016). Blockchain es un sistema libre de confianza, a prueba de manipulaciones, auditable y autorregulador, que no requiere intervención humana para ejecutar la computación (Atzori, 2017). Esta base de datos cifrada, que sirve como un depósito de información irreversible e incorruptible, permite, por primera vez, a personas no relacionadas llegar a un consenso sobre la realización de una transacción o evento en particular sin la necesidad de una autoridad de control (Wright y De Filippi, 2015).

Las soluciones basadas en Blockchain se están desarrollando en la actualidad en muchas áreas por grandes corporaciones de tecnología informática como IBM, Microsoft (Azure), Intel, etc., pero también por nuevas empresas que están creciendo rápidamente. Según Grech y Camilleri (2017), la mayoría de los Estados miembros de la Unión Europea probablemente experimenten con tecnologías Blockchain. Algunos están trabajando en estrategias nacionales, mientras que otros están llevando a cabo ensayos de aplicación específica. Entre estos países, se encuentran Estonia, Holanda, etc.

\subsection{Ventajas y desventajas de la tecnología Blockchain}

Las nuevas tecnologías siempre plantean debates, especialmente cuando estas se aplican al mundo financiero, que está constantemente en el punto de mira de escándalos y 
crisis. Por esta razón, es importante establecer cuáles son los beneficios y los inconvenientes de las cadenas de bloques.

A primera vista puede parecer una tecnología muy complicada que hace mover el dinero, $\mathrm{y}$, por tanto, si analizamos los métodos tradicionales de pago, se puede ver que cada transacción se registra en bases de datos privadas que son propiedad de entidades bancarias o estatales (Atzori, 2017).

Estas bases de datos están cerradas, lo que significa que no son accesibles al público en general. Normalmente, solo una entidad está a cargo de ellas, lo que se puede convertir en un problema. De hecho, se pueden producir estafas y, a veces, son atacadas por un hacker, que pone en peligro la red.

La tecnología Blockchain registra todas las transacciones en una moneda digital, no permite realizar pagos repetidos y es autentificada por muchas personas. La cadena de bloques también es descentralizada, lo que implica que, si una parte recibe un ataque, la red seguirá funcionando sin problemas (Nakamoto, 2008).

\subsection{Blockchain: una tecnología a prueba de manipulaciones}

Una de las principales ventajas de la tecnología Blockchain es que no se puede manipular. De hecho, cada bloque que se agrega a la cadena lleva una referencia encriptada del bloque anterior. Esta referencia es parte del problema matemático que debe resolver para añadir el siguiente bloque a la red y a la cadena (Nakamoto, 2008). Parte de resolver este problema matemático consiste en resolver números aleatorios denominados «nonce».

El nonce, combinado con otros datos, como el tamaño de la transacción, crea la huella digital denominada «hash». Esto está encriptado, por lo que lo hace seguro. Cada hash es único y debe cumplir con determinadas condiciones criptográficas. Una vez que esto pasa, el bloque se completa y se agrega a la cadena de bloques. Para manipular esto, que el bloque se añade al anterior, se necesita utilizar la criptografía como un rompecabezas; sin esto sería imposible realizarlo (Nakamoto, 2008).

Al mismo tiempo, la tecnología Blockchain presenta desventajas. De hecho, la red descentralizada no solo genera ventajas, sino que también crea problemas. Uno de los problemas más destacados es la cantidad de tiempo y costes que requiere una transacción (Wright

Uno de los problemas más destacados es la cantidad de tiempo y costes que requiere una transacción y De Filippi, 2015).

Además, cuando se crean nuevas monedas, generalmente están en manos de una menor cantidad de mineros. Los mineros son los que realizan en sí las operaciones, los 
que vigilan los nodos de forma más pasiva. Los mineros trabajan 24 horas al día/7 días a la semana para resolver problemas informáticos a cambio de una retribución. Si un minero controla más del $50 \%$ de la potencia minera de una moneda digital, teóricamente podría falsificar el libro de contabilidad de bloques (Nakamoto, 2008).

\section{Los mineros trabajan \\ 24 horas al dia/7 dias a \\ la semana para resolver problemas informáticos a cambio de una retribución}

Hay otros dos grandes problemas a los que la tecnología Blockchain debe enfrentarse:

- La presencia de material ilícito dentro de la cadena de bloques de la moneda digital. De hecho, cada bloque de la cadena de bloques contiene datos que hacen posible la transacción. Sin embargo, en algunos casos, los investigadores han encontrado material ilícito dentro de estos datos. Este material ilícito es muy difícil de encontrar, ya que está cifrado junto con los datos legales de la moneda digital.

- El tema de la recompensa. La forma en que se recompensa a los mineros por resolver los complejos problemas matemáticos que hacen que la transacción se realice es a través de la moneda digital. Se puede tener en cuenta que el proceso de minería detrás de la transacción puede ser extremadamente costoso, ya que el ordenador necesita una gran cantidad de energía para ejecutar este proceso; por lo tanto, la recompensa debe establecerse de tal manera que a los mineros les resulte rentable seguir realizando su trabajo.

\subsection{Beneficios de la tecnología Blockchain en educación}

La tecnología Blockchain tiene posibilidades ilimitadas y podría convertirse en una parte importante de los sistemas educativos.

Algunos de los beneficios de adoptar la tecnología Blockchain en el campo de la educación son los siguientes:

- Descentralización. Considerar una arquitectura distribuida P2P sobre una centralizada mejora la tolerancia a los fallos bizantinos al eliminar los puntos centrales de los fallos y los cuellos de botella (Veena, 2015).

- Escalabilidad. Permite la eliminación de situaciones en las que una o varias entidades controlan el almacenamiento y el procesamiento de información de un gran número de personas. 
- Fiabilidad. La información puede permanecer sin cambios, inmutable y distribuida a lo largo del tiempo en Blockchain. Cualquier participante del sistema puede verificar la autenticidad de los datos y asegurarse de que no hayan sido alterados (Reyna, Martín, Chen, Soler y Díaz, 2018).

- Seguridad. La información y las comunicaciones pueden ser seguras si se consideran transacciones de Blockchain (Prisco, 2016) basadas en protocolos criptográficos; por lo tanto, por ejemplo, Blockchain ofrece el potencial de hacer que los registros de títulos sean más seguros.

- Reducción de costes. Por último, pero no menos importante, los beneficios que pueden tener los centros de educación en la adopción de soluciones basadas en Blockchain incluyen la reducción de los costes administrativos y de la burocracia.

\section{2. ¿Qué es una ICO?}

En este apartado se da el siguiente paso hacia el objetivo de estudio del caso. De hecho, explicaremos qué es una ICO y cómo funciona.

En la actualidad, más y más nuevas empresas están construyendo sus negocios con tecnologías Blockchain y, en lugar de financiar sus negocios a través de las formas tradicionales, como pasa con las entidades financieras o la financiación de capital riesgo, están recurriendo a las criptomonedas.

Una ICO es una oferta inicial de moneda, y los datos muestran que, en los últimos años, esta forma de financiar negocios ha ido en aumento. Básicamente, una ICO es un nuevo método de financiación para nuevas empresas en el que se emiten nuevas monedas digitales (Schueffel, 2017).

\section{1. ¿Cómo funciona una ICO?}

En este apartado, resumiremos cómo funciona esta nueva manera de recaudar fondos. En primer lugar, la empresa crea una nueva moneda digital o una criptomoneda a través de las plataformas existentes, como Bitcoin o Ethereum. Entonces, la empresa puede lanzar la ICO. En esta etapa, los inversores minoristas pueden comprar los tokens digitales recién creados que pagan con otras criptomonedas, como bitcoins o ethers. Un token digital es un activo digital que está implementado dentro de la Blockchain de una criptomoneda (Reyna et al., 2018). 
Lo importante es tener en cuenta que esta forma de recaudar fondos podría ser sustancialmente diferente de la financiación con acciones. De hecho, incluso si el proceso de emitir una nueva criptomoneda y luego venderla a los inversores puede sonar muy parecido a la apariencia de una acción, una ICO puede ser significativamente diferente.

\subsubsection{Diferencias entre la ICO y una OPV}

Para aclarar lo comentado, este apartado se dedica a describir las principales diferencias entre una oferta pública de venta (OPA) y una ICO.

En primer lugar, una ICO es la creación de una nueva moneda digital en una cadena de bloques que luego se distribuye a través de un libro público. Una OPV es la emisión y distribución de acciones recién emitidas a inversores a través de bancos de inversión que trabajan como suscriptores.

Al definir los procesos, se puede observar una diferencia importante entre los dos procesos de financiación: la presencia de entidades financieras de inversión como suscriptores de la OPV que no existe en la ICO.

En segundo lugar, una OPV no es fácilmente disponible para la empresa. Solo las empresas establecidas pueden intentar recaudar fondos a través de una OPV. Es un proceso caro y el banco de inversión podría cobrar una comisión de hasta el $37 \%$ de las gestiones realizadas en la OPV, dependiendo del tamaño de la empresa y de cuánto esfuerzo debe realizar el banco para vender las nuevas acciones (Schueffel, 2017).

Algunas ICO están disponibles para todo tipo de startups y el producto mínimo viable (minimum viable product [MVP]) se reduce a documentos técnicos.

Por último, probablemente la mayor diferencia entre los dos métodos de financiación es la recompensa que obtiene el inversionista al comprar el nuevo token o acción emitida. En el caso de la OPV, los inversores pueden comprar las acciones de la empresa. Esto significa que cada inversor que compra acciones está comprando parte de la propia empresa. Cuando se trata de una ICO, el inversor puede comprar monedas digitales que se pueden usar para comprar un producto o un servicio dentro del ecosistema de la cadena de bloques.

Aunque existe la posibilidad de que el token digital se aprecie o se deprecie a través del tiempo, esto abre la posibilidad de cambiarlo por un beneficio o una pérdida. Según Bloomberg, las ICO recaudaron 22 billones de dólares estadounidenses en 2018 (en el cuadro 1 se muestra una lista de las mayores ICO realizadas). 
Cuadro 1. Lista de las mayores inversiones en ICO (datos en millones de dólares estadounidenses)

\begin{tabular}{l|l|l|l}
$\begin{array}{l}\text { Nombre de la ICO } \\
\text { Importe } \\
\text { recaudado }\end{array}$ & Nombre de la ICO & $\begin{array}{c}\text { Importe } \\
\text { recaudado }\end{array}$ \\
$\begin{array}{l}\text { 1. Filecoin } \\
257 \text { millones }\end{array}$ & 6. Status & 108 millones \\
\hline 2. Tezos & 232 millones & 7. QASH & 105 millones \\
3. EOS & 180 millones & 8. Aragon & 73 millones \\
4. SIRIN Labs Token & 157,9 millones & 9. Bankex & 70,6 millones \\
5. Banco & 153 millones & 10. TRON & 70 millones \\
\hline
\end{tabular}

Fuente: Coinist.

Hasta este momento hemos hablado de las ICO denominadas «tokens de utilidad». Dependiendo de su función, los tokens pueden clasificarse como tokens de utilidad y tokens de seguridad o valor. Es importante especificar esto por las siguientes razones:

- La empresa que analizaremos en este caso utiliza tokens de utilidad para su ICO.

- El tipo de emisión que se emite afecta en gran medida al proceso de la ICO. En particular, los tokens de utilidad se definen como un derecho prepago para consumir los bienes o servicios de un emisor. Un ejemplo de esto es el acceso al software o una cuenta en una plataforma.

Los tokens de seguridad, en cambio, deben contabilizarse siguiendo la regulación de cualquier valor de inversión, como acciones, bonos u otros productos de inversión. Si utilizáramos los tokens de seguridad, entonces la ICO sería muy parecida a la OPV y las diferencias entre los dos métodos de financiación descritos no se aplicarían.

\subsection{Ventajas y riesgos de lanzar una ICO respecto a otras fuentes de financiación}

Las ICO son un instrumento de financiación con muchas ventajas. Exponemos algunas de ellas: 
- Rapidez y facilidad. Cualquier empresa puede iniciar una ICO a través de varias plataformas, como Ethereum (Stellar Development Foundation [SDF], 2018).

- Acceso a mercados internacionales y online. El mercado potencial lo forman todas aquellas personas que tengan acceso a internet. La ICO se puede promocionar a través de redes sociales, webs especializadas y foros (Stellar Development Foundation y Luxembourg House of Financial Technology [SDF y LHoFT, 2018]).

- Poder de descentralización de la tecnología Blockchain. Esta tecnología influye en los procesos de la ICO. Además, al eliminar a los intermediarios, los recursos necesarios para completar cada transacción son mucho menores (SDF, 2018).

- Liquidez. Para los inversores, una de las grandes ventajas es que los mercados de criptomonedas suelen tener mucha liquidez. Es decir, se pueden adquirir grandes sumas de capital en poco tiempo (SDF y LHoFT, 2018).

- Democratización de la inversión. Los inversores no tienen que formar parte de un selecto grupo para poder invertir. El acceso a los tokens es público y se puede comprar desde cualquier punto del mundo (SDF, 2018).

Pero no todo son ventajas a la hora de invertir en una ICO. Casi todas las ventajas que acabamos de analizar tienen también su desventaja respecto al resto de fuentes de financiación. Estos son algunos de los riesgos de una ICO:

- Poca seguridad para el inversor. Casi nunca existen indicios reales de la tecnología o el negocio que desarrolla la empresa. En muchos casos no es más que un proyecto que puede funcionar o no (SDF y LHoFT, 2018).

- Incertidumbre alrededor del valor de los tokens. Existe tanta expectación ante los posibles beneficios de una ICO que, en ocasiones, el valor de los tokens reside más en la demanda potencial que en el producto real (SDF, 2018).

- Volatilidad del mercado. Existe una alta volatilidad en el mercado de las criptomonedas y de los tokens, ya que es una fuente de especulación de inversores. Así, las inversiones en tokens y criptomonedas requieren atención permanente (SDF y LHoFT, 2018).

- Desconocimiento de los inversores. La empresa que lleva a cabo las ICO nunca conoce la identidad real del inversor. Esto abre la puerta a que organizaciones ilegales muevan su dinero a través de la compra de tokens (SDF, 2018).

- Cambios inminentes en la legalidad. Apenas existen normativas regulatorias de las criptomonedas y los tokens, lo que no quiere decir que no se esté sujeto a regulaciones (SDF y LHoFT, 2018). 


\section{El caso de la IEBS Business School}

El caso a través del cual mostraremos cómo funciona la ICO y qué tipo de empresas pueden recurrir a este tipo de financiación es el de la IEBS Business School. Se trata de una escuela de negocios completamente online que ofrece formación en español y portugués. Su propósito es crear oportunidades iguales para todos, incentivando la motivación por aprender y desarrollar habilidades. Las características principales de la formación en la IEBS Business School son las siguientes:

- Aprender a medida que se hace un caso práctico.

- Sesiones online con expertos en el sector del programa.

- Interacción con profesionales en el sector del programa.

La idea que subyace detrás de la IEBS Business School tuvo su origen cuando los fundadores observaron que existía un problema en el sistema educativo y pensaron en resolverlo. Esta es la razón por la que los directivos que se encuentran al frente de la IEBS Business School afirman estar creando una revolución en la educación. Quieren explotar la tecnología de la cadena de bloques para certificar los conocimientos de los alumnos y, en una segunda fase, capacitar a los profesores para aumentar la motivación de los alumnos durante el aprendizaje.

\subsection{El problema y la solución propuesta}

Como se ha comentado anteriormente, la idea de la IEBS Business School es solucionar algunos problemas del sector educativo. Los problemas son los siguientes:

- No tener acceso a buenos maestros. Muchas personas no disponen de los medios financieros para pagar una educación de alta calidad; por lo tanto, un alto porcentaje de la población mundial acaba recibiendo una educación de baja calidad o no tiene acceso a una educación en absoluto.

- El aprendizaje en las clases es aburrido. Muchos estudiantes no aprovechan al máximo su experiencia de aprendizaje porque a veces las actividades en el aula son estandarizadas y aburridas. Esto resulta un desincentivo para que los estudiantes aprendan.

- Desconexión entre la teoría y las habilidades prácticas. A veces, la educación se centra demasiado en la teoría y deja a los estudiantes sin habilidades prácticas.

- Las personas no están motivadas para aprender. A pesar de que internet ofrece muchas herramientas y oportunidades para aprender, las personas parecen no sentirse motivadas. 
Teniendo en cuenta los problemas explicados, la IEBS Business School proporciona las siguientes soluciones:

- Ingeniería de gamificación del e-learning.

- Incentivos basados en el mérito para estudiantes y profesores (token de conocimiento).

- Interacción directa con el profesor.

- Prueba de registros inmutables para los profesores (una de las características de la tecnología Blockchain).

- Integración de terceros: contenido premium, profesores, anunciantes y patrocinadores que utilizan la infraestructura Blockchain.

La figura 1 muestra cómo funcionará el modelo de negocio de la IEBS Business School a través de una infraestructura Blockchain:

Figura 1. Modelo económico tokenizado

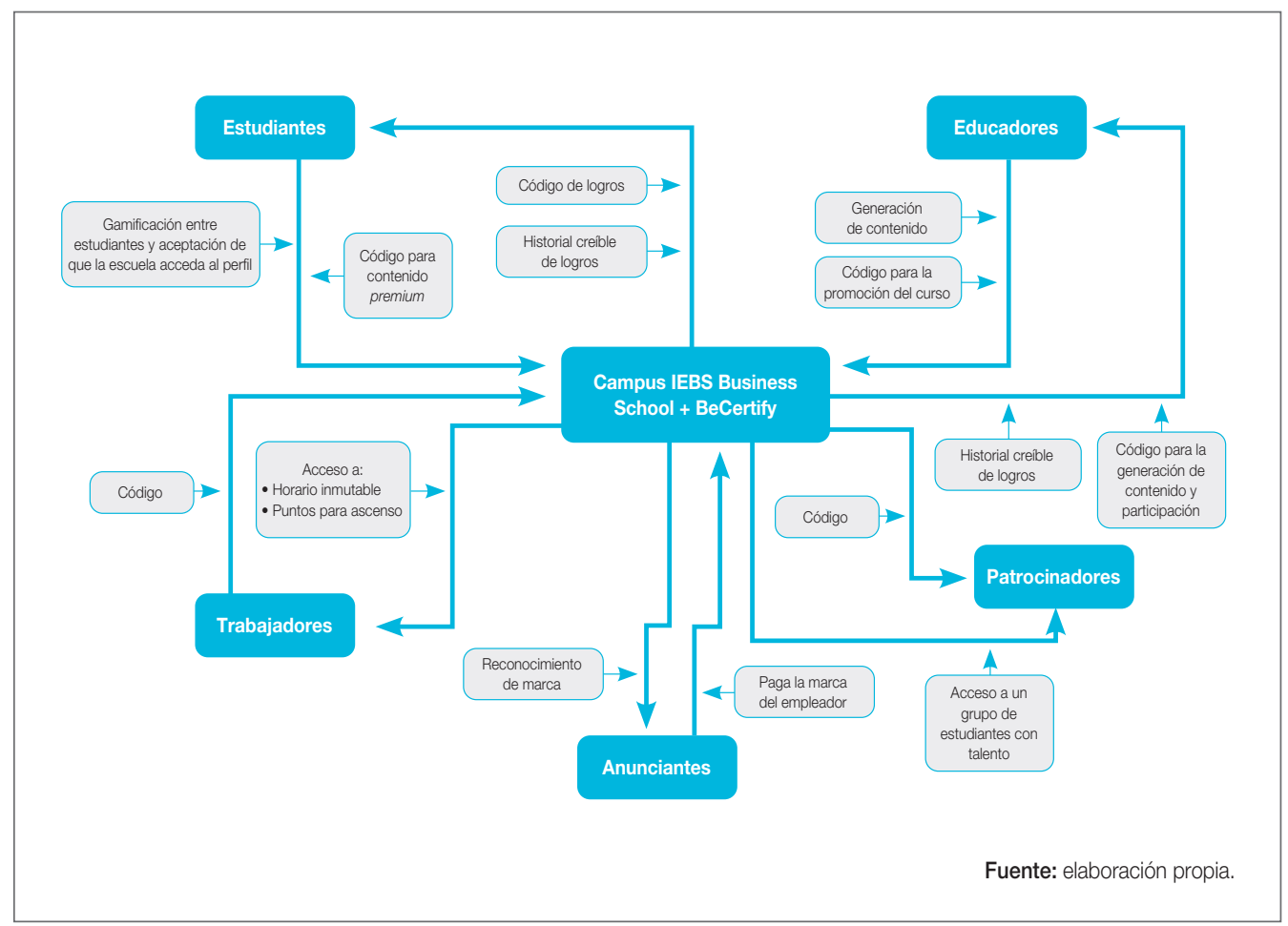


La figura 1 intenta explicar al lector cómo es el modelo de negocio de la empresa en una infraestructura Blockchain. En resumen, plantea irrumpir en el sector a través de mejorar sus servicios, utilizando la tecnología Blockchain y creando un sistema de incentivos que recompense a los mejores profesores y a los mejores estudiantes. El objetivo principal de este caso de estudio no es profundizar en el modelo de negocio, sino que el lector entienda el potencial de este proyecto. La plataforma se impulsa, a través de la cadena de bloques, a cinco elementos esenciales: estudiantes, profesores, trabajadores, anunciantes y patrocinadores. Cada una de estas categorías se beneficia de usar la plataforma de la siguiente manera:

- Alumnos. Recibirán tokens pagados para aprender, se beneficiarán de minutos de aprendizaje, tendrán un historial de logros y obtendrán comentarios sociales tanto de otros estudiantes como de profesores, además de tener acceso al trabajo. Se crean oportunidades a través de la red de networking.

- Profesores. Recibirán tokens pagados para enseñar y, a través de su historial personal, podrán convertirse en maestros estrella. Al mismo tiempo, también podrán obtener tokens por ayudar a personas con menos recursos.

- Trabajadores. Su principal ventaja proviene de su acceso a los registros y a los logros del campus de formación. Podrán seguir los logros de potenciales candidatos e incluso construir programas de fomento.

- Anunciantes. Podrán crear reconocimiento de marca por apoyar un programa o varios programas de la IEBS Business School.

- Patrocinadores. Pueden patrocinar talentos individuales o cursos del catálogo disponible.

\subsection{Roadmap de la IEBS Business School}

En dicho cuadro se describen los principales pasos relacionados con el código de desarrollo del talento, así como las expectativas de futuro que tiene la IEBS Business School.

En el cuadro 2 se puede analizar una estimación de la progresión de implantación de las diferentes etapas planteadas en el proyecto de puesta en marcha de la tecnología Blockchain, ya que, según los retrasos o inconvenientes encontrados en el proceso, los tiempos se verán alterados.

En agosto del 2019 se han estado realizando diferentes pruebas con la nueva plataforma Blockchain en Ethereum, a través del distribuidor de tecnología Blockchain BeCertify, para certificar los títulos y se han encontrado algunos inconvenientes que los técnicos de las dos empresas intentan solucionar para llegar a la implantación definitiva en el ERP (enterprise resource planning) interno de la IEBS Business School. 
Cuadro 2. Previsión de implantación de la tecnología Blockchain en la IEBS Business School

\begin{tabular}{l|l}
\multicolumn{1}{c|}{ Fecha } & \multicolumn{1}{c}{ Descripción } \\
\hline Mayo 2019 & $\begin{array}{l}\text { Se inició el desarrollo de la plataforma Blockchain en Ethereum a través del } \\
\text { distribuidor de tecnología Blockchain BeCertify para certificar títulos. }\end{array}$ \\
\hline Septiembre 2019 & Generación de tokens con ayuda de BeCertify. \\
\hline Febrero 2020 & Versión prototipo del campus virtual de tecnología Blockchain de IEBS. \\
\hline Mayo 2020 & Versión 1: completa para el campus virtual de tecnología Blockchain de IEBS. \\
\hline Noviembre 2020 & Inicio del desarrollo de la versión 2 del campus virtual en tecnología Blockchain. \\
\hline Futuro & $\begin{array}{l}\text { Desarrollo de nuevas funcionalidades para empresarios, patrocinadores y B2B. } \\
\text { Nuena asociación estratégica e integración de terceros que mejoren la expe- }\end{array}$ \\
\hline
\end{tabular}

Fuente: elaboración propia.

\subsection{Venta de tokens}

Como parte de la ICO, la IEBS Business School tiene que crear una nueva moneda digital y venderla al público. Por lo tanto, este punto describe brevemente la venta de tokens de la IEBS Business School y proporciona información adicional en relación a la misma.

La IEBS Business School emitirá un token compatible con Ethereum ERC20 con la utilidad central de ser parte del sistema que recompensará a los usuarios del campus y que se usará para pagar los servicios dentro del mismo. Quien comparta la visión de la IEBS Business School podrá comprar el token digital recién creado, que estará disponible durante un periodo de venta privado, preventa y venta colectiva. Habrá limitaciones mínimas y máximas para permitir que todos participen. También existe el «límite flexible», que se calcula como el nivel mínimo de inversión para asegurar el desarrollo del campus en tecnología Blockchain. La IEBS Business School proporcionará un número máximo de 30.000.000 de tokens para la venta. El valor de un token será de 0,10 euros, calculado como equivalente en ethers, que se fijará una vez que se inicie la preventa. La figura 2 muestra los detalles que se han explicado, pero vale la pena establecer un soft cap de 1.000 .000 de euros, lo que corresponde a la cantidad mínima de dinero que una criptomoneda, en el caso de la 
IEBS Business School, puede recibir de los inversores en su ICO. Si este objetivo no se cumple, el dinero se devuelve a los inversores. Se puede ver que un hard cap es el objetivo financiero del token.

Figura 2. Mapa de la ICO de la IEBS Business School

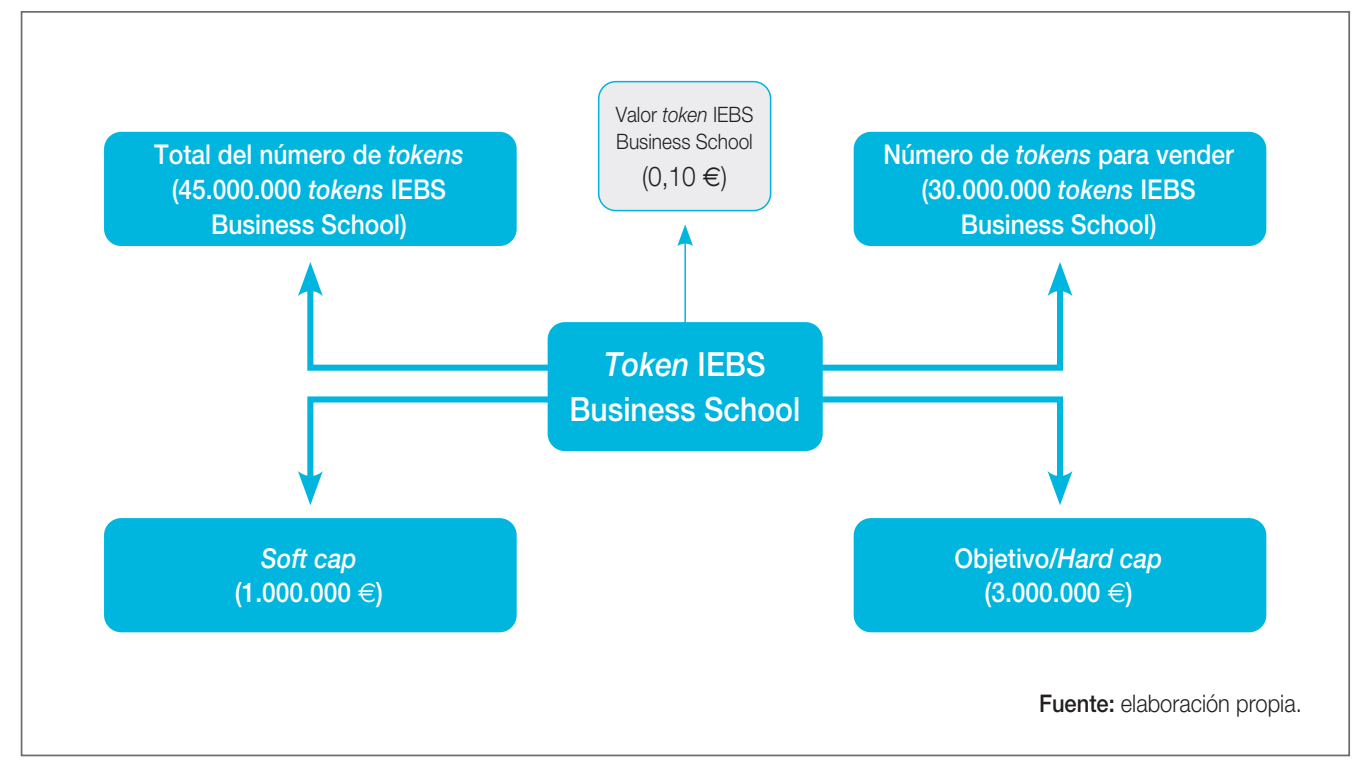

\subsubsection{Distribución del token de la IEBS Business School}

Además de los detalles relacionados con la venta de los tokens de la IEBS Business School, también se ha pensado en la redistribución de los tokens. Como se puede ver en la figura 2, más de la mitad de los tokens disponibles estarán a la venta, mientras que el resto se redistribuirán entre el equipo, el campus, las asesores y las recompensas. Para mayor claridad, las recompensas son unos incentivos para que un grupo de participantes difunda el proyecto. Los tokens del equipo (12\%) se administrarán de la siguiente forma:

- El $50 \%$ de los tokens de la IEBS Business School estarán disponibles al mismo tiempo que para el público.

- El $25 \%$ de los tokens de la IEBS Business School estarán disponibles durante seis meses.

- El resto de tokens de la IEBS Business School estarán disponibles hasta un año. 
Este enfoque para el periodo de bloque se ve como una señal del equipo de la IEBS Business School hacia un compromiso a largo plazo. El uso y el bloque del $100 \%$ de los tokens de la IEBS Business School de los asesores se realizará en un periodo de 12 meses. La preventa, también denominada «evento generador de token» (token generation event [TGE]), comenzará el 15 de noviembre de 2019 y finalizará el 31 de enero de 2020.

\section{La importancia de la tecnología Blockchain para la IEBS Business School}

La IEBS Business School es una de las escuelas de negocio online más importantes de España. Esta característica la hace estar en una buena posición respecto al e-learning de los competidores. En primer lugar, la IEBS Business School quiere proporcionar recompensas basadas en el mérito tanto para los estudiantes como para los profesores. El campus va a utilizar un conjunto de puntos de recompensas para asignar incentivos de forma regular. Por lo tanto, la IEBS Business School proporcionará un sistema de incentivos de token. En segundo lugar, pondrá a disposición de los estudiantes un campus de aprendizaje habilitado en tecnología Blockchain. El formulario podrá reconocer el perfil de los alumnos y agregar un itinerario de aprendizaje personalizado y eficiente, compuesto de diferentes clases de distintos profesores. Estas características garantizan a la IEBS Business School una ventaja competitiva dentro del sector de la educación online. Por último, ninguno de los competidores de la IEBS Business School utiliza una tecnología basada en Blockchain.

La IEBS Business School se basa en que la formación e-learning de educación superior necesita una organización dotada de derechos para garantizar la identidad de sus estudiantes. El primer desafío que se desea alcanzar es mantener la privacidad y la seguridad de los datos almacenados digitalmente. La tecnología Blockchain puede ser programada para conservar de forma virtual todo tipo de información, comenzando por los certificados, hasta registros académicos, las deudas de los estudiantes y todo lo que se pueda codificar. La Blockchain planteada por la IEBS Business School es una infraestructura de clave pública (public key infrastructure [PKI]) para establecer una plataforma segura. La clave pública es una forma avanzada de criptografía asimétrica en la que los usuarios tienen dos claves que no realizan las mismas funciones: una permite encriptar la información y la otra permite desencriptar la información. Estas claves son asimétricas.

El desafío al que la IEBS Business School quiere hacer frente es la validez de los datos. En un momento en el que la información es bastante abundante, veloz y cambiante, ser capaz de verificar cualquier solicitud es cada vez más importante. Y el último desafío que se propone es el tiempo de consulta de la información. 


\section{Conclusiones}

La IEBS Business School es un ejemplo interesante de cómo las nuevas tecnologías están incorporándose en el mundo financiero y reestructurando la forma de aprender. De hecho, a lo largo de este análisis, y de la introducción de los conceptos de «Blockchain» y de «criptomonedas», se ha explicado el mecanismo de una ICO. Este método es diferente de la financiación tradicional y está creciendo a medida que pasa el tiempo. Los reguladores están tratando de entender cómo establecer, de manera correcta, las principales directrices para esta nueva tecnología. Sin embargo, es importante darse cuenta de cómo la creatividad y el capital humano están adquiriendo más importancia en la sociedad actual y, especialmente, en el mundo empresarial. La IEBS Business School es un ejemplo de empresa que, con solo presentar una idea apoyada por profesionales preparados, podría explotar una tecnología innovadora para intentar tener éxito en el sector de la educación; algo que no se nos habría pasado por la cabeza hace unos años. Esta es la prueba de que la tecnología Blockchain está dando forma no solo a sectores tecnológicos, sino también a sectores más tradicionales y conservadores, como es el financiero o el educativo.

Por tanto, la IEBS Business School quiere evolucionar hacia una nueva pedagogía, ya que, mientras la sociedad valore las acreditaciones existentes y los estudiantes paguen para conseguir estas acreditaciones en instituciones reconocidas antes que buscar alternativas, entonces, universidades o escuelas de negocio presenciales tendrán una oportunidad. La acreditación y el prestigio están basados en su eficacia como institución en la que aprender. Las instituciones que afrontan los nuevos modelos se convierten en más efectivas y en entornos de aprendizaje más deseables.

Con esto, la IEBS Business School quiere que el aprendizaje online libere capital intelectual, permitiendo que el estudiante, en el campus, dedique su tiempo no solo a absorber información, sino también a pensar e investigar. Podríamos definir una «clase» como un proceso en el que los conocimientos del profesor se trasladan a los apuntes del estudiante sin pasar por un procesamiento cerebral. Esto no es lo que se necesita en la era digital para una nueva generación de estudiantes. A los estudiantes actuales les gusta hablar mientras aprenden, les gusta compartir, inmersos en la tecnología digital, les gusta probar cosas nuevas, a menudo de forma rápida, etc. Quieren que su proceso de enseñanza sea divertido e interesante, y desean experimentar el placer de descubrir las cosas por ellos mismos. Muchos adolescentes dedican una gran cantidad de su tiempo a navegar por internet y a leer sobre diferentes ideas, lejos de la cultura existente en ese momento, y piensan que es útil todo lo que aprenden por sí mismos, debatiendo ideas con otras personas en los foros.

La IEBS Business School, a través de su Blockchain, permite que la formación del siglo XXI se convierta en una red y en un ecosistema colaborativo. En realidad, los inno- 
vadores, como la IEBS Business School, tienen entre sus manos la gran oportunidad de crear una experiencia mejor para estudiantes de cualquier parte del planeta, uniendo los mejores materiales de aprendizaje en línea y permitiendo a los estudiantes organizar sus itinerarios de aprendizaje con el soporte de una red de instructores y facilitadores educativos, algunos de los cuales pueden ser locales, y otros, de cualquier parte del mundo. Con la finalidad de hacer posible estos objetivos, la IEBS Business School ha puesto en marcha profundos cambios estructurales y ha estrechado sus relaciones con los docentes. La IEBS Business School, a través de su Blockchain, proporciona una plataforma en la que crear una red global para el aprendizaje.

Aparte de lo comentado, el modelo planteado por la IEBS Business School se puede aplicar a otros casos de empresas similares en los siguientes puntos:

- Establecer el intercambio de contenidos, donde los profesores compartan ideas y suban sus materiales a internet para que otros los utilicen libremente.

- La coinnovación en el contenido, cuando los docentes colaboran más allá de las fronteras de la institución para cocrear nuevos materiales de enseñanza, utilizando elementos como las wikis y otras herramientas.

- La institución se convierte en un nodo en la red global de docentes, estudiantes e instituciones, aprendiendo de forma colaborativa.

Muchas instituciones de formación tienen un problema con la idea de la educación como negocio. Se puede plantear aplicar la tecnología Blockchain para reducir los costes de formación de los estudiantes gracias a la acreditación a través de modelos de pago por éxito. La Blockchain provee elementos para conseguir el objetivo de reducir los costes de formación:

- Mecanismo fiable para confirmar que los estudiantes que se apunten a las clases completen efectivamente el curso, hagan los exámenes y dominen la materia.

- Un mecanismo de pago.

- Contratos inteligentes que pueden convertirse en planes de aprendizaje.

Funciona como si fuera una organización de microfinanciación donde los estudiantes pueden registrar su progreso. Los donantes pueden pagar a los estudiantes de forma individual, asignar importes económicos a objetivos de aprendizaje y pagar de acuerdo a los logros conseguidos. Se puede automatizar el proceso de aprendizaje a través de un test en línea donde la tecnología Blockchain puede confirmar la identidad del estudiante y registrar el progreso antes de pagar los fondos; es decir, establecer una cartera inteligente para la educación. 


\section{Referencias bibliográficas}

Atzori, M. (1 de mayo de 2017). Blockchain governance and the role of trust service providers: the TrustedChain ${ }^{\circledR}$ network. SSRN. Disponible en <https://papers.ssrn.com/ sol3/papers.cfm?abstract_id=2972837> (consultado el 3 de mayo de 2019).

Carr, N. G. (2003). It doesn'tmatter. Harvard Business Review. Disponible en <https://motamem.org/wp-content/uploads/2017/02/ nicholas-carr-it-does-not-atter.pdf> (consultado el 15 de mayo de 2019).

Grech, A. y Camilleri, A. F. (2017). Blockchain in education. Luxembourg: Publications Office of the European Union 2017, 132 S. JRC Science for Policy Report. Disponible en <https://www.pedocs.de/voll texte/2018/15013/pdf/Grech_Camilleri _2017_Blockchain_in_Education.pdf> (consultado el 10 de febrero de 2019).

Filipowski, A. (2018). Blockchain for 2018 and beyond: a (growing) list of Blockchain use cases. Medium. Disponible en $<$ https://medium.com/fluree/blockchainfor-2018-and-beyond-a-growing-list-ofblockchain-use-cases-37db7c19fb99> (consultado el 14 de abril de 2019).

lansiti, M. y Lakhani, K. R. (2017). The truth about Blockchain. Harvard Business Review. Disponible en <https://enterprisers project.com/sites/default/files/the_truth_ about_blockchain.pdf> (consultado el 2 de mayo de 2019).

Jaime Maestre, R. (2018a). Blockchain en el sector de la logística: trazabilidad y transparencia. IEBS. Disponible en <https:// www.iebschool.com/blog/blockchainlogistica/> (consultado el 30 de mayo de 2019).

Jaime Maestre, R. (2018b). Blockchain rompe las cadenas musicales de Youtube, Spotify y los grandes sellos discográficos. IEBS.
Disponible en <https://www.iebschool.com /blog/blockchain-cadenas-musicales-you tube-spotify-discograficos-tecnologia/> (consultado el 22 de mayo de 2019).

Jaime Maestre, R. (mayo de 2019). La Blockchain revolucionarà la comunicació de l'empresa. Comunicació: Revista de Recerca i d'Anàlisis, 36(1). Disponible en $<$ http://revistes.iec.cat/index.php/TC/arti cle/view/145793> (consultado el 22 de mayo de 2019).

Kharpal, A. (16 de julio de 2018). Tokenization: the world of ICOs. CNBC. Disponible en $<$ https://www.cnbc.com/2018/07/13/ini tial-coin-offering-ico-what-are-they-howdo-they-work.html> (consultado el 13 de abril de 2019).

Mattila, J. y Seppälä, T. (13 de agosto de 2015). Blockchains as a path to a network of systems. ETLA, 45. Disponible en $<$ https://www.etla.fi/wp-content/uploads/ ETLA-Raportit-Reports-45.pdf> (consultado el 14 de mayo de 2019).

Malviya, H. (10 de diciembre de 2016). How Blockchain will defend IOT. SSRN. Disponible en <http://dx.doi.org/10.2139/ssrn. $2883711>$ (consultado el 14 de marzo de 2019).

Mougayar, W. (2016). The Business Blockchain: Promise, Practice and Application of the Next Internet Technology. Nueva Jersery, EE. UU.: Wiley.

Nakamoto, S. (2008). Bitcoin: a peer-to-peer electronic cash system. Bitcoin. Disponible en <https://bitcoin.org/bitcoin.pdf> (consultado el 3 de febrero de 2019).

Piscini, E., Guastella, J., Rozman, A. y Nassim, T. (24 de febrero de 2016). Blockchain: democratized trust: distributed ledgers and the future of value. Deloitte University Press. Disponible en <http://dupress.com/ 
articles/blockchain-applications-and-trustin-a-global-economy/> (consultado el 18 de mayo de 2019).

Prisco, G (2015). Slock.it to introduce smart locks linked to smart Ethereum contracts, decentralize the sharing economy. Bitcoin Magazine. Disponible en $<$ https://bitcoinmagazine.com/articles/ slock-it-to-introduce-smart-locks-linkedto-smart-ethereum-contracts-decentra lize-the-sharing-economy-1446746719> (consultado el 24 de mayo de 2019).

Preukschat, A. (Coord.). (2017). Blockchain: la revolución industrial de internet. Madrid: Planeta de Libros.

Reyna, A., Martín, C., Chen, J., Soler, E. y Díaz, M. (2018). On blockchain and its integration with loT. Challenges and opportunities. Future Generation Computer Systems, 88, 173-190. Disponible en <https://doi. org/10.1016/j.future.2018.05.046> (consultado el 27 de mayo de 2019).

Schueffel, P. (15 de diciembre de 2017). Alternative distributed ledger technologies Blockchain vs. Tangle vs. Hashgraph-A high-level overview and comparison. SSRN. Disponible en <https://ssrn.com/abstract=3144241> (consultado el 21 de abril de 2019).

SDF. (17 de julio de 2018). Stellar first DLT to obtain sharia certification for payments \& asset tokenization. Stellar Blog. Disponible en <https://www.stellar.org/blog/stellar-re ceives-sharia-compliance-certification- transfers-tokenization> (consultado el 11 de abril de 2019).

SDF y LHoFT. (2018). Understanding initial coin offerings: technology, benefits, risks and regulation. The LHoFT. Disponible en <https:// www.Ihoft.com/en/the-future-of-fintech-lieshere/understanding-initial-coin-offeringstechnology-benefits-risks-and-regulation> (consultado el 10 de abril de 2019).

Tapscott, D. y Tapscott, A. (2016). Blockchain Revolution: How the Technology Behind Bitcoin Is Changing Money, Business, and the World. Nueva York, EE. UU.: Penguin Random House.

Torpey, K. (2018). Microsoft to embrace decentralized identity systems built on bitcoin and other Blockchains. Forbes. Disponible en <https://www.forbes.com/sites /ktorpey/2018/02/12/microsoft-to-embra ce-decentralized-identity-systems-builton-bitcoin-and-other-blockchains/> (consultado el 14 de marzo de 2019).

Veena, P. (2015). Device democracy: saving the future of the internet of things. IBM Corporation. Disponible en <https://www. $\mathrm{ibm}$.com/downloads/cas/Y5ONA8EV> (consultado el 4 de abril de 2019).

Wright, A. y Filippi, P. de. (10 de marzo de 2015). Decentralized Blockchain technology and the rise of lex cryptographia. SSRN. Disponible en <https://ssrn.com/ abstract $=2580664>$ (consultado el $30 \mathrm{de}$ abril 2019). 


\title{
Metodología personalizada con resultados de aprendizaje garantizados
}

La formación de lenguas extranjeras se lleva a cabo dentro de la normativa vigente de la enseñanza de idiomas en España y en la Comunidad Europea. Los cursos presentan un diseño adaptado al Marco Común Europeo de Referencia para las Lenguas y a la metodología propia de la UDIMA: un sistema de enseñanza cercano, flexible, actual, dinámico y personalizado.

\section{Cursos generales}

\section{Cursos universitarios de español}

Títulos propios no oficiales que tratan de acercar al alumno a las habilidades lingüísticas necesarias para el dominio del español como lengua extranjera.

\section{Curso de chino (nivel iniciación)}

Este curso permite que los estudiantes adquieran las capacidades necesarias para defenderse en situaciones sencillas de la vida cotidiana y para desenvolverse socialmente en el idioma chino. Además, prepara al alumno para el examen oficial de primer nivel HSK1/A1.

\section{Cursos de preparación de exámenes}

\author{
Certificate in Advanced English (CAE)
}

La finalidad del Certificate in Advanced English (CAE) es proporcionar a estudiantes y profesores de idiomas, en una variedad de situaciones, el acceso a una amplia gama de exámenes internacionales de gran calidad, test y diplomas para profesores que les ayuden a lograr sus metas personales y que repercutan favorablemente en su experiencia de aprendizaje y desarrollo profesional. El Certificate in Advanced English (CAE) se corresponde con el nivel C1 del Marco de Referencia Europeo.

\section{Curso de preparación TKT CLIL Module (TKT)}

Este curso, indicado para profesores que están preparando el examen TKT CLIL, les aportará las herramientas y el conocimiento necesarios para superar el examen oficial y para mejorar el proceso de enseñanza-aprendizaje en el aula CLIL. El curso se dará en inglés, ayudando al alumno a reforzar el vocabulario, la gramática y las funciones que han sido aprendidos previamente con explicaciones y correcciones puntuales. Los contenidos se centrarán en los temas necesarios para dar una clase CLIL (principios teóricos, planificación en el aula, técnicas y evaluación).

\section{Preliminary English Test (PET)}

El Preliminary English Test (PET) es un examen de inglés de nivel intermedio. La preparación de este examen ayudará a mejorar las destrezas lingüísticas y el uso del inglés para comunicarse con hablantes nativos de manera cotidiana. El Preliminary English Test (PET) se correponde con el nivel B1 del Marco de Referencia Europeo.

\section{First Certificate in English (FCE)}

La finalidad del First Certificate in English (FCE) es proporcionar a los estudiantes de idiomas la preparación suficiente que les permita lograr sus metas personales, repercutiendo favorablemente en su experiencia de aprendizaje y desarrollo profesional. El First Certificate in English (FCE) se corresponde con el nivel B2 del Marco de Referencia Europeo. 